\title{
Impact de l'application de trois pesticides dans le potentiel de colonisation du rhizobium sur deux accessions de Niébé en Côte d'Ivoire
}

\author{
Clovis Boni N'dodo KOFFI ${ }^{1}$, Marie Hélène Ahébé KOFFI ${ }^{2 *}$, Marie N'Guettia YAH ${ }^{3}$ et \\ Diallo Hortense ATTA ${ }^{4}$ \\ ${ }^{1,2,3}$ Laboratoire d'Amélioration de Production Végétale, Université Jean Lorougnon GUEDE (UJLoG), BP \\ 150 Daloa, Côte d'Ivoire. \\ ${ }^{4}$ Laboratoire de Phytopathologie, Université Nangui Abrogoua (UNA), 02 BP 801 Abidjan 02, Côte d'Ivoire. \\ *Auteur correspondant; E-mail: ahebemarie77@yahoo.fr; Tel: (+225) 07489911
}

\section{RESUME}

Le Niébé (Vigna unguiculata) est une légumineuse alimentaire qui fournit plus de la moitié des protéines consommés. Cependant, sa culture est sujette à l'attaque de nombreux pathogènes et les rendements restent faibles. Une des alternatives pour un meilleur rendement est l'utilisation des produits agrochimiques. L'objectif recherché dans cette étude est d'optimiser la production de Vigna unguiculata. Pour atteindre cet objectif, des expérimentations ont été entreprises pour évaluer l'effet de l'utilisation du Furadan, du Mancomax et du Forum sur la nodulation lors de la culture de deux accessions ( $N b n K$ et $\mathrm{NnB}$ ) de niébé. Pour ce faire, le sol de culture provenant d'une jachère de 2 ans au sein de l'université a été traité avec le Furadan 3 jours avant le semis tandis qu'un traitement foliaire a été effectué avec le Mancomax, et Forum à la dose du fabricant après la levée des plants de 5 jours. Un second traitement a été fait trois semaines après le semis. Les résultats montrent que les trois fongicides testés réduisent significativement de 50 à $84 \%$ le nombre de nodules des deux variétés et les nodules de l'accession $\mathrm{NnB}$ sont plus réduits que celles de l'accession $\mathrm{NbnK}$.

(C) 2019 International Formulae Group. All rights reserved

Mots clés : Effet, nodulation, produits agrochimiques, Vigna unguiculata,

\section{Impact of application of three pesticides on potential of nodulation of two cowpea accession in Côte d'Ivoire}

\begin{abstract}
Nibaceous (Vigna unguiculata) is a food pulse that provides more than half of the protein consumed. However, its culture is subject to attack by many pathogens and yields remain low. One of the alternatives for better yield is the use of agrochemicals. The aim of this study is to optimize the production of Vigna unguiculata. To achieve this aim, experiments were undertaken to assess the effect of the use of Furadan, Mancomax and Forum on nodulation the culture of two accessions (NbnK and $\mathrm{NnB}$ ) of nibaceous. For this purpose, the culture soil from a 2-year fallow within the university was treated with Furadan 3 days prior to seedling, while leaf treatment was performed with Mancomax, and Forum at the manufacturer's dose after the 5-day seedling was lifted. A second treatment was done three weeks after the seedling. Results show that the three fungicides tested significantly reduce the number of nodules in both varieties by $50-84 \%$ and that the nodules of $\mathrm{NnB}$ accession are smaller than those of $\mathrm{NbnK}$ accession.
\end{abstract}

(C) 2019 International Formulae Group. All rights reserved

Keywords: Effect, nodulation, agrochemicals, Vigna unguiculata. 


\section{INTRODUCTION}

La sécurité alimentaire est aujourd'hui un enjeu majeur de développement de la Côte d'Ivoire. Face à la croissance démographique et à la demande alimentaire, sans cesse croissante l'on est contraint de se tourner vers les cultures vivrières entre autre les légumineuses à graines, dont le niébé (Vigna unguiculata) longtemps marginalisé (N'gbesso, 2013). Le niébé est une importante culture dans les régions tropicales et subtropicales notamment en Afrique subsaharienne. Il représente une importante source de revenus pour les paysans. Les jeunes feuilles, les gousses et les graines constituent des sources de protéine (21-25\%), de vitamines et d'élément minéraux pour l'alimentation humaine et animal (Ndiaye, 2007 ; Dugje et al., 2009; Moussa et al., 2011). Sa culture contribue également à la restauration de la fertilité des sols grâce à son haut potentiel de fixation biologique de l'azote atmosphérique par les populations microbiennes au nombre desquelles est compté les bactéries nodulantes (Graham et Vance, 2003). Egalement, cette plante est adaptée à un large éventail de sols et de conditions d'humidité et permet de diminuer le développement des mauvaises herbes fréquemment observées dans les rotations culturales (Gilbert et al., 2010). Malgré la valeur nutritive et l'importance agronomique reconnue à cette légumineuse, les rendements demeurent très faibles. La hausse des prix des denrées alimentaires au cours de ces dernières liée aux changements climatiques ayant pour conséquence, la pauvreté du sol, la rareté de la pluviométrie et les contraintes parasitaires seraient des causes de la baisse de rendement (Kinimo, 2013). Selon le Fonds des Nations Unies pour la population, cette baisse de rendement accentue la vulnérabilité des ménages entrainant une hausse du nombre de personnes sous-alimentées à environ 795 millions de personnes dans le monde (FIDA, 2011). Ainsi, la sécurité alimentaire devient l'un des grands enjeux du développement des pays africains, et en particulier pour les moins avancés d'entre eux tel que la Côte d'Ivoire. Pour pallier à ce problème, les producteurs utilisent les herbicides et divers fongicides au cours du cycle de production. Cependant, bien que l'usage des produits agrochimiques soit largement répandu, le rendement escompté ne suit pas. Alors, l'espoir mis dans l'utilisation d'engrais chimiques pour y remédier s'avère décevant et d'importantes menaces de survie des bactéries nodulantes par l'utilisation de pesticides sont signalées par des études. C'est le cas des investigations menées par Konaté et al. (2015) qui ont montré que l'application d'herbicide à l'occurrence le glyphosate réduisait la densité de populations de bactéries nodulantes. Cependant, certaines études réalisées tendent à montrer que les pesticides n'ont aucun effet sur les populations de rhizobia. En effet, Dubey et al. (2012) ont conclu que le Furadan n'avait aucun impact sur les bactéries nodulantes en Inde. Pour eux les bactéries dégraderaient aisément les molécules de Furadan. Aynalem et Assefa (2017) ont également rapporté que l'utilisation de glyphosate et de Mancomax en Ethiopie n'entravait pas la nodulation et les performances de fixation de l'azote par les rhizobia. Au regard de toutes ces observations, l'objectif assigné à cette étude est d'améliorer le rendement de deux accessions de Niébé. Il a consisté à l'évaluation de trois pesticides dans le potentiel de colonisation du rhizobium sur le Niébé en Côte d'Ivoire.

\section{MATERIEL ET METHODES Matériel végétal}

Le matériel végétal utilisé dans cette étude a été constitué de graines de deux variétés de niébé. Ces variétés sont issues de la collection de l'Université Jean Lorougnon Guédé de Daloa. Les différents codes attribués à ces variétés ont été faits sur la base du numéro de l'accession, la provenance des graines, la couleur de l'enveloppe tégumentaire et la forme des graines. Il s'agit des accessions NbnK (Niébé noir-blanc collectée à Korhogo) et $\mathrm{NnB}$ (Niébé noire collectée à Biankouman), respectivement à la Figure 1 et Figure 2. Ces deux variétés sont de cycle court et se sont distinguées par leurs rendements élevés au sein de la collection. 


\section{Matériel chimique}

Pour la réalisation de cette étude, trois pesticides ont été utilisés, à savoir Mancomax (mancozèbe), Forum (cuivre+dimethomorphe) et Furadan (carbofuran). Le choix de ces fongicides est basé sur leur grand usage par les producteurs et leur disponibilité sur le marché local. Les spécifications concernant ces produits agrochimiques sont consignées dans le Tableau 1.

\section{Méthode \\ Préparation de la culture du niébé}

L'expérience a été faite à la température ambiante $\left(\approx 25^{\circ} \mathrm{C}\right)$ du laboratoire. Pour chaque accession de niébé, vingt sachets de culture ont été utilisés par traitement. L'expérience a été répétée trois fois. Chaque sachet $\left(\approx 1,15 \mathrm{dm}^{3}\right)$ a été rempli avec un échantillon de sol (sans fertilisation chimique et organique) prélevé de la jachère de l'université. Ces échantillons de sol ont été tamisés à $2 \mathrm{~mm}$ et pesés à l'aide d'une balance. La quantité de sol fine ayant servi au remplissage des sachets est de $1,5 \mathrm{~kg}$. Les différents sols ont été humidifiés avec environ 0,5 à 1 litre d'eau par sachet avant le semis. Le semis a été fait $24 \mathrm{~h}$ après l'humidification à raison de 2 graines de niébé par sachet. Cinq jours après la levée des plantules. Chaque accession de niébé a subi deux traitements avec chaque pesticide.

\section{Evaluation de l'effet du Furadan sur le nombre moyen de nodules des plantes de niébé.}

Les graines de niébé ont subi un premier traitement, avec les granules de Furadan pur, 3 jours avant le semis. Trois semaines après le semis, une seconde application avec le même pesticide a été faite. A cet effet, 3,6 g de granule de Furadan pur ont été incorporés au sol autour des pieds de niébé. Un traitement témoin sans apport de Furadan a été également fait avec chaque accession.
Evaluation de l'effet du Mancomax et de Forum sur le nombre moyen de nodules des plantes de niébé

Le premier traitement avec le Mancomax et le Forum a été fait 5 jours après la levée. Trois semaines après le semis, $3,33 \mathrm{~g}$ de chaque produit ont été dilués séparément avec 11 d'eau pour obtenir les solutions de traitement. Ensuite, $50 \mathrm{ml}$ de chaque solution a servi à traiter les feuilles des plants de niébé avec un pulvérisateur manuel de capacité 1 litre. Un traitement témoin sans apport de Mancomax et de Forum a été également fait avec chaque accession.

\section{Collectes des données}

La collecte des données a été effectuée à un mois deux semaines soit 45 jours après la levée. Elle a consisté d'abord, à une forte humidification du sol avec environ 0,5 à 1 litre d'eau par sachet pour éviter que les racines contenant les nodules restent dans le sol lors du déracinement des plantules du niébé. Ensuite, les plantules de chaque lot ont été déterrées délicatement et les nodules de chaque plantule ont été comptés sous une loupe binoculaire.

Enfin le taux de réduction des nodules de chaque accession est obtenu selon la formule suivante :

$$
T R(\%)=\frac{N N P}{T E} * 100 \text { avec NNP }=\mathrm{TE}-\mathrm{NNAT}
$$

TR $(\%) \quad$ : Taux de réduction des nodules

TE : Nombre de nodulation normale ou témoin sans traitement

NNAT : Nombre de nodule après traitement

NNP : Nombre de nodule perdu à cause du traitement

\section{Analyses statistiques}

L'analyse de la variance (ANOVA) à deux critères de classification a été utilisée pour évaluer l'effet des pesticides sur les moyennes des nodules des deux accessions de Niébé. Le test de Newman-Keuls a servi à la comparaison des moyennes présentant des différences significatives au seuil de 5\%. Le logiciel STATISTICA Version 7.1 a été utilisé. 


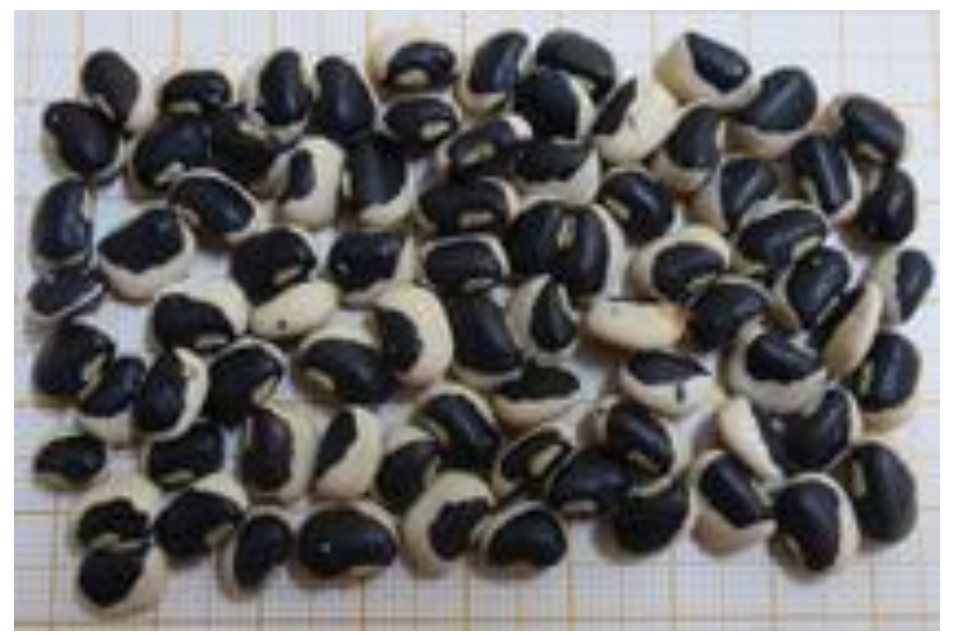

Figure 1 : Accession noir-blanc (NbnK) de niébé provenant de Korhogo (Côte d' Ivoire).

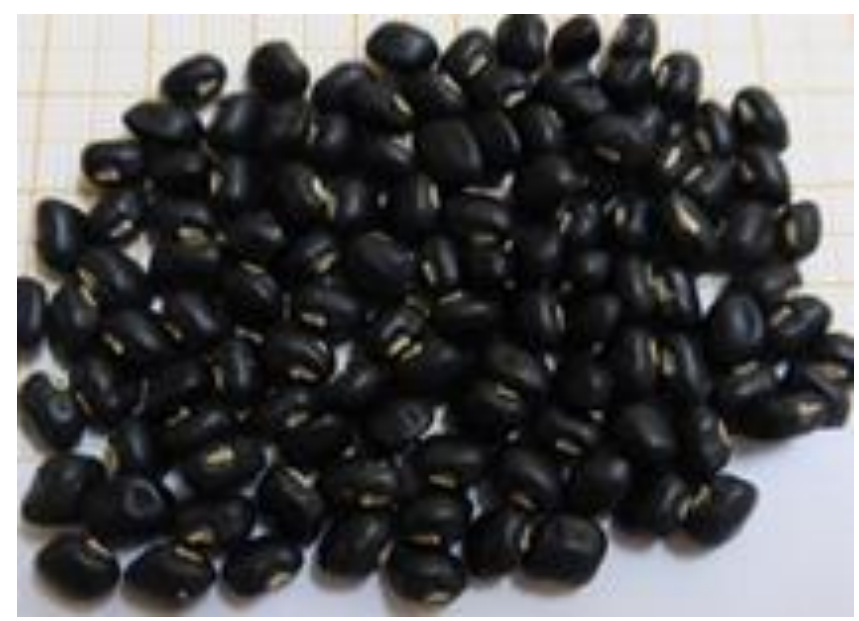

Figure 2 : Accession noire $(\mathrm{NnB})$ de niébé provenant de Biankouman (Côte d' Ivoire).

Tableau 1 : Noms commerciaux et spécifications techniques des pesticides utilisés.

\begin{tabular}{lllll}
\hline $\begin{array}{l}\text { Type de } \\
\text { pesticide }\end{array}$ & $\begin{array}{l}\text { Nom } \\
\text { commercial }\end{array}$ & Molécule active & Formulation & $\begin{array}{l}\text { Dosage / } \\
\text { Dose d'application }\end{array}$ \\
\hline Nématicide & Furadan & $\begin{array}{l}\text { 2,3-dihydro-2,2-dimethyl-7- } \\
\text { benzofuranyl methylcarbamate } \\
\text { (Carbamate) }\end{array}$ & granulé & $\begin{array}{l}3 \% \mathrm{de} \text { Furadan pur } \\
4 \mathrm{~kg} / \mathrm{ha} \mathrm{soit} 0,4 \mathrm{~g} / \mathrm{m}^{2}\end{array}$ \\
& & Mancozèbe & Poudre \\
Fongicide & Mancomax & mouillable & $\begin{array}{l}800 \mathrm{~g} / \mathrm{kg} \text { Mancozèbe } \\
3,33-6,67 \mathrm{~g} / \mathrm{l}\end{array}$ \\
& 80WP & Dimethomorphe + cuivre & $\begin{array}{l}\text { Poudre } \\
\text { mouillable }\end{array}$ & $\begin{array}{l}60 \mathrm{~g} / \mathrm{kg} \\
\text { Dimethomorphe } \\
400 \mathrm{~g} / \mathrm{kg} \text { Cuivre }\end{array}$ \\
\hline Fongicide/ & Forum & & & \\
Bactéricide & & & & \\
& & & &
\end{tabular}




\section{RESULTATS}

\section{Impact du Furadan sur la nodulation des accessions de niébé}

L'analyse statistique du tableau 2 a montré que l'effet du Furadan a très significativement réduit le nombre de nodules par plant de niébé et par accession $(\mathrm{P}<0,0001)$. Le traitement au Furadan de la rhizosphère des plants de niébé de l'accession de Biankouman à réduit la nodulation de $72 \%$. Ainsi, les nodules de l'accession $\mathrm{NnB}$ (Biankouman) passent d'environ 21 nodules pour le témoin à environ 6 nodules. Quant à l'accession NbnK de Korhogo, la réduction de la nodulation est estimée à plus de $84 \%$. En effet, le nombre de nodules par plant passe de 81 (témoin) à environ 13 lorsqu'on applique le Furadan. Les résultats montrent que la nodulation de l'accession $\mathrm{NnB}$ est plus faible que celle de l'accession NbnK lorsqu'aucun pesticide n'est appliqué. L'effet du Furadan sur la nodulation de l'accesssion $\mathrm{NnB}(72 \%$ de réduction) est moins important que celui sur l'accession NbnK ( $84 \%$ de réduction).

\section{Impact du Mancomax et du Forum sur la nodulation des accessions de niébé}

L'analyse statistique du tableau 2 a montré que l'effet du Mancomax et du Forum a très significativement réduit le nombre de nodules par plant de niébé et par accession $(\mathrm{P}<0,0001)$. L'application du Mancomax et du Forum sur les plants de niébé collectés à
Biankouman $(\mathrm{NnB})$ montre une réduction très importante de la capacité de nodulation de cette accession. En effet, le traitement de l'accession $\mathrm{NnB}$ au Mancomax réduit de $70,64 \%$ le nombre de nodules par rapport au témoin (100\%)). Sur la même accession, la réduction du nombre de nodules avec le Forum est statistiquement identique $(54,72 \%)$ à celle du Mancomax. Au niveau de l'accession $\mathrm{NbnK}$ les fongicides utilisés ont réduit différemment le nombre de nodules. Une réduction plus importante de la nodulation a été notée avec l'application du Forum. En effet, le Forum a réduit de $68,61 \%$ le nombre de nodules par rapport au témoin (81 nodules/pied). Par contre, une réduction de $51,45 \%$ de la nodulation a été observée avec l'application du Mancomax.

\section{Effet de l'interaction variété (accession)- traitement}

Bien que le nombre de nodules des plants varie selon la variété (accession) et selon le traitement, l'interaction variététraitement a influencé très significativement le potentiel de nodulation selon l'analyse statistique (Tableau 2). La nodulation de l'accession $\mathrm{NbnK}$ est fortement réduite à l'application du Furadan ( $84 \%$ de réduction) par rapport à l'accession $\mathrm{NnB}(72 \%)$. Par contre lorsqu'on applique le Mancomax ou le Forum c'est l'effet inverse qui est observé.

Tableau 2: Incidence du Carbofuran (Furadan), du Cuivre + Dimethomorphe (Forum) et du Mancozèbe (mancomax) sur la nodulation de deux accessions de Niébé.

\begin{tabular}{lllll}
\hline Accession & Traitement & $\begin{array}{l}\text { Nombre moyen de nodules/plant } \\
( \pm \text { F }\end{array}$ & P \\
\hline NnB & Tf & $5,65 \pm 0,59^{\mathrm{a}}$ & & \\
NnB & Tm & $6,01 \pm 1,41^{\mathrm{a}}$ & & \\
NnB & Te & $9,27 \pm 4,35^{\mathrm{a}}$ & 19,80 & $<0,0001$ \\
NbnK & Tf & $12,67 \pm 2,47^{\mathrm{ab}}$ & & \\
NnB & Te & $20,48 \pm 2,21^{\mathrm{ab}}$ & \\
NbnK & Te & $25,5 \pm 6,5^{\mathrm{b}}$ & \\
NbnK & Tm & $39,45 \pm 3,33^{\mathrm{c}}$ & \\
NbnK & Te & $81,26 \pm 5,18^{\mathrm{d}}$ & \\
\hline
\end{tabular}

Les valeurs portant la même lettre dans la même colonne sont statistiquement identiques (Test de Newman-keuls, $\alpha=5 \%$ NnB: Niébé noir Biankouma; NbnK: Niébe blanc noir de Khorogo ; Tm: traitement au Mancozèbe (Mancomax) ; Te: temoin Tc: traitement au cuivre + Dimethomorphe ; Tf: Traitement au Carbofuran (Furadan). 


\section{DISCUSSION}

Les nombres de nodule par plant des accessions $\mathrm{NnB}$ et $\mathrm{NbnK}$ ont été significativement réduit après application du Furadan. Cependant, la nodulation de l'accession $\mathrm{NnB}$ est plus faible que celle de l'accession NbnK. Aussi, l'application du Mancomax et du Forum sur les plants de niébé collectés à Biankouman $(\mathrm{NnB})$ et Korhogo $(\mathrm{NbnK})$ a réduit la capacité de nodulation de ces accessions. Cependant, le nombre de nodules avec le Forum et le Mancomax sur l'accession $\mathrm{NnB}$ est statistiquement identique, alors qu'avec l'accession $\mathrm{NbnK}$, ce nombre est plus réduit avec le Mancomax. Cette importante réduction du potentiel de nodulation du niébé de l'accession Nnb dans notre étude pourrait être expliquée par la période et le mode d'application des différents pesticides. En effet, lors de notre étude les accessions de niébé ont reçu deux applications distinctes de chaque pesticide, selon les périodes. Ainsi, le Furadan a été appliqué au semis et à trois semaines après le semis, tandis que le Forum et le Mancomax ont été appliqués 5 jours après la levée et trois semaines après le semis. Ces périodes d'application pourraient correspondre à la période de nodulation dans le stade de développement du niébé entrainant ainsi un impact significatif sur la nodulation. Les travaux de Dubey et al. (2012) ont montré que le mode d'application couplé à la dose d'application des pesticides réduit les populations de rhizobia. Aussi, cette baisse de nodulation pourrait indiquer que les bactéries associées à la nodulation sont sensibles à ce pesticide.

Encore, la réduction du nombre d'inoculum au niveau des variétés de niébé pourrait être dû au fait que les plantules n'aient pas reçu une inoculation bactérienne. Ce constat corrobore les travaux de Légère (2009), Haro et al. 2015 et Brahimaet al. (2018). Ceux-ci ont affirmé dans leurs différentes études, que l'inoculation bactérienne des graines de soja permet d'obtenir un rendement élevé grâce à la fixation symbiotique de l'azote. Contrairement à cette affirmation, Ama-Abina et al. (2012) soutiennent que l'inoculation bactérienne n'a pas d'influence sur les variétés de soja testées. Selon eux, les plants de soja disposent suffisamment d'azote mis à leur disposition par les bactéries, en plus de l'azote déjà disponible dans le sol pour satisfaire leurs besoins trophiques.

La réduction du nombre de nodules des accessions pourrait être due au fait que nos travaux aient été conduit dans les sachets. Traoré et al. (2008), montre que l'utilisation des sachets à volume réduit conduit à l'épuisement rapide des nutriments, à un mauvais développement racinaire dû au manque d'espace et à la mauvaise aération. En effet, le déficit d'éléments minéraux conduit à une importante réduction $\mathrm{du}$ nombre d'organes fructifères formés, quel que soit la variété ou le cultivar de soja, avec un rendement de moins d'une tonne à l'hectare chez les producteurs (N'Gbesso et al., 2017). Ainsi, une nutrition azotée des plants de soja assurée par les bactéries Rhizobium pourrait accroître leur productivité (N'Gbesso et al., 2010). Le manque d'apport de phosphate pourrait également être la cause de la réduction des nodules. Ouandaogo et al. (2016) dans leur étude ont montré que l'apport combiné du phosphate avec le compost ou le fumier entraine une hausse de la nodulation du niébé.

La nodulation est significativement réduite par le cuivre contrairement aux Mancomax. Ces résultats vont dans le sens des observations faites par Cevheri et al. (2011) dans leur étude portant sur la tolérance des isolats de nodules racinaires aux métaux lourds. Selon eux, l'application des doses recommandées de Mancomax et de Cuivre inhibe la nodulation. Cela pourrait, d'une part, être dû au fait que le cuivre soit associé au Dimethomorphe et d'autre part à l'usage relativement récente des pesticides contenant le cuivre dans le vivrier en Côte d'Ivoire.

Aussi, selon ces mêmes auteurs, les fongicides inhibent la nodulation en affectant les enzymes cellulolytiques et pectolytiques, essentiels pour la pénétration racinaire, sécrétés par les rhizobia. De plus, Tindwa et Msumali (2010) ont montré que le niébé et le soja étaient plus sensibles au cuivre in vitro et in vivo que le haricot commun. L'étude menée par Küçük et Cevheri (2012) sur la tolérance des rhizobia aux métaux lourds et aux pesticides a montré que la plupart des souches se révèle résistante au Zinc et aux antibiotiques tandis que certaines sont sensibles à la Pénicilline G et au Kanamycine. 
Aussi, plusieurs micropopulations de bactéries pourraient être impliquées dans la nodulation des légumineuses et pourraient avoir une intensité de fixation variable selon les variétés. Ainsi, la baisse de la nodulation constatée dans cette étude pourrait expliquer la sensibilité de certaines micropopulations vis-à-vis des pesticides. Ces résultats sont contraire à ceux obtenus par Das et de Mukherjee (2000) dans leur étude portant sur l'action des insecticides dans la transformation microbienne. Ces auteurs ont montré que les pesticides n'ont aucune influence significative sur la microflore du sol et que certaines bactéries pourraient les dégrader.

\section{Conclusion}

L'objectif de cette étude était d'évaluer trois pesticides dans le potentiel de colonisation du rhizobium sur le Niébé en Côte d'Ivoire. Il ressort de cette étude que l'application $\mathrm{du}$ Furadan réduit significativement la nodulation des plants de l'accession $\mathrm{NnB}$ par rapport à celle de l'accession NbnK. De plus, le nombre de nodule après application du Forum et du Mancomax sur l'accession $\mathrm{NnB}$ est statistiquement identique, alors qu'avec l'accession $\mathrm{NbnK}$, ce nombre est plus réduit avec le Mancomax. En somme, cette étude doit se poursuivre et déterminer la diversité des rhizobia et l'impact de ces différentes micropopulations dans la fixation de l'azote atmosphérique avant et après les fortes doses de pesticides.

\section{CONFLIT D'INTERETS}

Les auteurs déclarent qu'il n'y a aucun conflit d'intérêts.

\section{CONTRIBUTIONS DES AUTEURS}

Ce travail a été conduit avec la collaboration de tous les auteurs. CBNK a conduit l'étude, collecté traité les données et écrit le premier jet du manuscrits. MHAK a rédigé et finalisé l'article. Les co-auteurs MNY et AHD ont lu et approuvé le manuscrit final.

\section{REMERCIEMENTS}

Les auteurs remercient les autorités de l'Université Jean Lorougnon Guédé (Côte d'Ivoire) et le groupe de recherche du
Laboratoire d'Amélioration de la Production Végétale de ladite Université.

\section{REFERENCES}

Ama-Abina J, Beugre GF, N'Gbesso MF, Brou ND, Yoro GR. 2012. Effets d'un herbicide et de l'inoculation sur les facteurs de rendement du soja cultivé sur un sol gravillonnaire de plateau. International Journal of Biological and Chemical Sciences, 6(5): 1970-1978. DOI: http://dx.doi.org/10.4314/ijbcs.v6i5.7

Aynalem B, Assefa F. 2017. Effect of glyphosate and mancozeb on the rhizobia isolated from nodules of Vicia faba $\mathrm{L}$. and on their $\mathrm{N}_{2}$-fixation North Showa, Amhara Regional State, Ethiopia. Advances in Biology, 1(1): 1-7. DOI: 10.1155/2017/5864598

Brahima B, Turquin L, N'GBESSO M. 2018. Effet de l'inoculum bactérien de la souche IRAT-FA 3 de Bradyrhizobium japonicum sur la production de trois variétés de soja en Côte d'Ivoire International Journal of Biological and Chemmical Science, 12(6): 2667-2679. DOI:

https://dx.doi.org/10.4314/ijbcs.v12i6.16

Cevehri C, Küçük Ç, Çetin E. 2011. Fungicide, antibiotic, heavy metal resistance and salt tolerance of root nodule isolates from Vicia palaestina. African Journal of Biotechnology, 10(13): 2423-2429. DOI: 10.5897/AJB10.1291

DAS AC, Mukharjee D. 2000. Influence of insecticides on microbial transformation of $\mathrm{N}$ and $\mathrm{P}$ in Typic orchraguarf soil, Journal of Agriculture, food Chemical, 48 (8): 3728-3732.

Dubey V, Singh D, Shukla A, Shukla S, Singh N. 2012. Effect of application of different pesticides to leguminous crops of microflora of Sidhi district (M.P.). International Journal of Engineering Research and Development, 3(12): 1-3.

Dugje YI, Omoigui OL, Ekelem F, Kamara YA, Ajeigbe H. 2009. Production du niébé en Afrique de l'Ouest: guide du paysan. Institut international d'agriculture tropicale (IITA), Ibadan, Nigeria, $20 \mathrm{p}$.

FIDA. 2015. L'État de l'insécurité alimentaire dans le monde. Objectifs internationaux 
2015 de réduction de la faim: des progrès inégaux, $2 \mathrm{p}$.

Gilbert L, Souapibe PS, Venasius L, Gnokreo F., djimasbeye N' Ndoutamia G. 2009. Efficacité de l'association des céréales et du niébé pour la production de grains et la lutte contre Striga hermonthica (Del.). Savanes africaines en développement, 8 p.

Grahan PH, Vance CP. 2003. Legumes: importance and constraints to greater use. Plant Physiology, 131(3): 872-877. DOI: https://doi.org/10.1104/pp.017004

Haro H, Sanon KB, Krasova-Wade T, Kane A, N'Doye I, Traoré AS. 2015. Réponse à la double inoculation mycorhizienne et rhizobienne du niébé (variété, KVX3964-5-2D) cultivé au Burkina Faso. International Journal of Biological and Chemical Sciences, 9(3): 1485-1493. DOI: http://dx.doi.org/10.4314/ijbcs.v9i3.31

Kinimo RY. 2013. Déterminants de la sousalimentation des ménages en côte d'ivoire : cas des régions centre et centre-est. European Scientific Journal, 9(14): 207-228.

Konate I, Amani K, Koffi M, Doumbia ML, Berraho EB, Filali-Maltouf A, Kouadio YJ. 2015. Influence of herbicides on symbiotic bacteria survival, growth and nodulation of legume niébé (Phaseolus vulgaris) in Center-West of Côte d'Ivoire. International Journal of Agriculture Innovations and Research, 4(2): 317-322.

Küçük Ç, Cevehri C. 2012. Tolerance of rhizobia isolated from Trifolium species in Southeast region, Șanliurfa, Turkey. African Journal of Agricultural Research, 7(10): 1462-1467. DOI: 10.5897/AJAR11.061

Légère A. 2009. Biodiversité et productivité utopie ou réalité? Constat après 18 années de pratiques de conservation des sols, 6p.

Moussa B, Lowenberg DJ, Fulton J, Boys K. 2011. The economic impact of cowpea research in West and Central Africa: a regional impact assessment of improved cowpea storage technologies. Journal Stored Product Research, 47: 147-156. DOI: $10.1016 /$ j.jspr.2011.02.001
Ndiaye M. 2007. Ecology and Management of Charcoal Rot (Macrophomina phaseolina) on Cowpea in the Sahel. $\mathrm{PhD}$ thesis, Wageningen University, the Netherlands, $114 \mathrm{p}$.

N'Gbesso MF, N'guetta ASP, Kouamé N, Foua BK. 2010. Évaluation de l'efficience de l'inoculation des semences chez 11 génotypes de soja (Glycine max (L.) Merril) en zone de savane de Côte d'Ivoire. Sciences et Nature, 7(1): 59-67. DOI: http://dx.doi.org/10.4314/scinat.v7i1.599 31

N'gbesso FM, Fondio L, Dibi BEK, Djidji HA, Kouamé CN. 2013. Etude des composantes du rendement de six variétés améliorées de niébé [Vigna unguiculata (L.) Walp]. Journal of Applied Biosciences, 63: 4754-4762. DOI: http://dx.doi.org/10.4314/jab.v63i1.8724 9

N'Gbesso MF, Fondio L, Coulibaly ND, Kouamé NC. 2017. Efficacité symbiotique de cinq souches locales de rhizobiums sur les paramètres de croissance du soja. International Journal of Biological and Chemical Sciences, 11(5): 2327-2340. DOI: http://dx.doi.org/10.4314/ijbcs.v11i5.30

Ouandaogo N, Ouattara B, Pouya BM, Gnankambary Z, Nacro BH, Sedogo PM. 2016. Effets des fumures organominérales et des rotations culturales sur la qualité des sols. International Journal of Biological and Chemical Science, 10(2): $\quad 904-918 . \quad$ DOI: http://dx.doi.org/10.4314/ijbcs.v10i2.37

Tindwa H, Msumali GP. 2010. Effect of soil copper accumulation on proliferation and survival of rhizobia. Second RUFORUM Biennal meeting rapport, Entebbe, Uganda, 713-717.

Traoré K, Toé AM. 2008. Capitalisation des initiatives sur les bonnes pratiques agricoles au Burkina Faso. DVRD/DPV/MAHRH, $99 \mathrm{p}$. 